\title{
Electric Conductivity of Hot and Dense Quark Matter in a Magnetic Field with Landau Level Resummation via Kinetic Equations
}

\author{
Kenji Fukushima \\ Department of Physics, The University of Tokyo, 7-3-1 Hongo, Bunkyo-ku, Tokyo 113-0033, Japan \\ Yoshimasa Hidaka \\ Theoretical Research Division, Nishina Center, RIKEN, 2-1 Hirosawa, Wako, Saitama 351-0198, Japan \\ and iTHEMS Program, RIKEN, 2-1 Hirosawa, Wako, Saitama 351-0198, Japan
}

(Received 19 November 2017; published 17 April 2018)

\begin{abstract}
We compute the electric conductivity of quark matter at finite temperature $T$ and a quark chemical potential $\mu$ under a magnetic field $B$ beyond the lowest Landau level approximation. The electric conductivity transverse to $B$ is dominated by the Hall conductivity $\sigma_{H}$. For the longitudinal conductivity $\sigma_{\|}$, we need to solve kinetic equations. Then, we numerically find that $\sigma_{\|}$has only a mild dependence on $\mu$ and the quark mass $m_{q}$. Moreover, $\sigma_{\|}$first decreases and then linearly increases as a function of $B$, leading to an intermediate $B$ region that looks consistent with the experimental signature for the chiral magnetic effect. We also point out that $\sigma_{\|}$at a nonzero $B$ remains within the range of the lattice-QCD estimate at $B=0$.
\end{abstract}

DOI: $10.1103 /$ PhysRevLett.120.162301

Introduction.-Extreme matter of quarks and gluons in quantum chromodynamics (QCD) could be realized as a quark-gluon plasma in nucleus-nucleus collisions and as quark matter in the neutron star cores. Nowadays, the collision experiment called the beam energy scan is aiming to explore the QCD phase diagram at finite temperature $T$ and quark chemical potential $\mu \lesssim T$. Interestingly, such hot and dense QCD matter may be exposed under a strong magnetic field $B$ if the nucleus-nucleus collision is noncentral. The presence of strong $B$ provides us with an ideal probe to topological contents of the QCD vacuum, as exemplified by the chiral magnetic effect (CME) [1].

To quantify topological effects induced by $B$, we need transport coefficients, among which the most important is the electric conductivity $\sigma$. The CME signature in a condensed-matter system of Weyl semimetals is the negative magnetoregistance, that is, the quadratic rise of $\sigma_{\mathrm{CME}}(B) \propto B^{2}[2]$, which has been first detected experimentally in Ref. [3] assuming that nontopological $\sigma$ is insensitive to $B$. In contrast to the condensed-matter system, for hot and dense quark matter, we can make a first-principles estimate for $\sigma(B)$ from QCD directly. Moreover, in the nucleus-nucleus collision, $\sigma(B)$ controls the lifetime of $B[4,5]$.

Published by the American Physical Society under the terms of the Creative Commons Attribution 4.0 International license. Further distribution of this work must maintain attribution to the author(s) and the published article's title, journal citation, and DOI. Funded by SCOAP.
So far, $\sigma(B)$ has been perturbatively calculated in QCD under a scale hierarchy, $\sqrt{e B} \gg T \gg g T$, where $e$ represents the charge of the proton and $g$ the QCD charge, using the lowest Landau level approximation (LLLA) [6,7]. Usually, the LLLA is a reasonable approximation for strong $B$ and has been adopted for various QCD observables such as the heavy quark diffusion constant [8], the bulk viscosity [9], etc. The validity of the LLLA is questionable, however, for $\sigma(B)$ involving ( $u$ and $d$ ) quarks with small mass $m_{q}$, i.e., $\sigma \rightarrow \infty$ as $m_{q} \rightarrow 0$, since the scattering phase space is too severely restricted by the approximation.

In the present work we significantly revise the calculation of $\sigma(B)$ in a different (more realistic) regime, $\sqrt{e B} \gg g T$, in which we neglect $T$-induced quark damping $\left(\sim g^{2} T\right)$, namely, $\Delta \varepsilon>e B / T \gg g^{2} T$, where $\Delta \varepsilon$ is an energy gap associated with adjacent Landau levels. Then, we will find that our $\sigma$ with full Landau level resummation shows a much milder $m_{q}$ dependence than the LLLA result. We will also see that the $B$ dependence is minor, which justifies comparing our finite- $B \sigma$ to the lattice-QCD measured value at $B=0$ [10-12].

Some definitions. - The electric conductivity is given by the following Kubo formula:

$$
\sigma^{i j}=\lim _{k_{0} \rightarrow 0} \lim _{k \rightarrow 0} \frac{1}{2 i k_{0}}\left[\Pi_{R}^{i j}(k)-\Pi_{A}^{i j}(k)\right],
$$

where $\Pi_{R / A}^{\mu \nu}(k)$ are the retarded and the advanced polarization functions, respectively, defined by $\Pi_{R / A}^{i j}(k):=$ $\pm i \int d^{4} x e^{i k \cdot x} \theta( \pm t)\left\langle\left[j^{i}(x), j^{j}(0)\right]\right\rangle$, where “+” is for $R$ 
and "-" is for $A$. We note that, for the conductivity used in the hydrodynamics, $j^{i}$ is not necessarily an electric current operator, $j_{\mathrm{em}}^{i}=\sum_{f} q_{f} \bar{\psi}_{f} \gamma^{i} \psi_{f}$, where $f$ refers to flavor and $q_{f}$ is the electric charge of the $f$ quark, i.e., $q_{u}=(2 / 3) e$ and $q_{d}=-(1 / 3) e$. We need to subtract the contribution from hydrodynamic modes, which can be done with a subtracted current operator $j^{i}=j_{\mathrm{em}}^{i}-n_{e} T^{0 i} /\left(\mathcal{E}+\mathcal{P}_{i}\right)$ with the electric charge density $n_{e}$, the energy momentum tensor (operator) $T^{\mu \nu}$, the energy density $\mathcal{E}=\left\langle T^{00}\right\rangle$, and the pressure $\mathcal{P}_{i}=\left\langle T^{i i}\right\rangle$ [13]. In the following we evaluate this correlation function involving the electric current and the energy momentum tensor (1) using perturbation theory and the Boltzmann equation with magnetic effects fully taken into account.

For perturbative calculations of $\prod_{R / A}^{i j}(k)$ we need the free quark propagator at finite $B$. The retarded propagator in the flavor $f$ sector is given by a sum over the Landau levels labeled by $n$ as

$S_{R / A}^{f}(p)=\sum_{n=0}^{\infty} \frac{-S_{n}^{f}(p)}{p_{0}^{2}-\varepsilon_{f n}^{2} \pm i \epsilon p_{0}}=\sum_{n=0}^{\infty} \frac{-S_{n}^{f}(p)}{p_{\|}^{2}-m_{f n}^{2} \pm i \epsilon p_{0}}$,

where the (flavored) Landau quantized energy dispersion is $\varepsilon_{f n}=\sqrt{p_{z}^{2}+2\left|q_{f} B\right| n+m_{f}^{2}}$ and we defined $m_{f n}^{2}:=$ $2\left|q_{f} B\right| n+m_{f}^{2}, p_{\perp}^{\mu}:=\left(0, p_{x}, p_{y}, 0\right)$, and $p_{\|}^{\mu}:=\left(p_{0}, 0,0, p_{z}\right)$. Here, we chose the Fock-Schwinger gauge, and the $B$ direction along the $z$ axis without loss of generality. The numerator $S_{n}^{f}(p)$ has Dirac index structures decomposed as

$$
\begin{aligned}
S_{n}^{f}(p)= & \left(\not \|_{\|}+m_{f}\right)\left[P_{+}^{f} A_{n}\left(4 \xi_{p}^{f}\right)+P_{-}^{f} A_{n-1}\left(4 \xi_{p}^{f}\right)\right] \\
& +\not p_{\perp} B_{n}\left(4 \xi_{p}^{f}\right)
\end{aligned}
$$

with $\xi_{p}^{f}:=\left|\boldsymbol{p}_{\perp}\right|^{2} /\left(2\left|q_{f} B\right|\right)$. We introduced $A_{n}(x):=$ $2 e^{-x / 2}(-1)^{n} L_{n}(x)$, and $B_{n}(x):=4 e^{-x / 2}(-1)^{n-1} L_{n-1}^{(1)}(x)$, where $L_{n}(x)=L_{n}^{(0)}(x)$ and $L_{n}^{(\alpha)}(x)$ represent the generalized Laguerre polynomials [14]. In the above expression $P_{ \pm}^{f}$ represents the projection operator $P_{ \pm}^{f}$ := $\left[1 \pm \operatorname{sgn}\left(q_{f} B\right) i \gamma^{1} \gamma^{2}\right] / 2$.

Electric conductivity.-Proceeding to the conductivity calculation, we decompose the anisotropic tensor structure of the electric conductivity using $\hat{B}^{i}:=B^{i} /|\boldsymbol{B}|$ as

$$
\sigma^{i j}=\sigma_{H} \epsilon^{i j k} \hat{B}^{k}+\sigma_{\|} \hat{B}^{i} \hat{B}^{j}+\sigma_{\perp}\left(\delta^{i j}-\hat{B}^{i} \hat{B}^{j}\right),
$$

where $\sigma_{H}$ represents the Hall conductivity for an electric current perpendicular to both electric and magnetic fields. In the $R / A$ basis, the polarization tensor at the one-loop order reads

$$
\begin{aligned}
\Pi_{R}^{\mu \nu}(k)= & -i \sum_{f} q_{f}^{2} \int \frac{d^{4} p}{(2 \pi)^{4}} \operatorname{tr}\left[\gamma^{\mu} S_{R R}^{f}(k+p) \gamma^{\nu} S_{A R}^{f}(p)\right] \\
& -i \sum_{f} q_{f}^{2} \int \frac{d^{4} p}{(2 \pi)^{4}} \operatorname{tr}\left[\gamma^{\mu} S_{R A}^{f}(k+p) \gamma^{\nu} S_{R R}^{f}(p)\right],
\end{aligned}
$$

apart from the hydrodynamic mode subtraction, which will be taken into account later. We can straightforwardly perform the integration (5) to get $\sigma_{H}=n_{e} / B$, which is nothing but the Hall conductivity. Up to the one-loop order $\sigma_{\perp}=0$, which is intuitively understood from the Landau quantization of transverse motion. A nonzero value of $\sigma_{\perp}$ appears from the two-loop and higher order contributions. Here, we give a parametric estimate, $\sigma_{\perp} / T \sim g^{2} T^{2} /|e B| \ll 1$ for $\sqrt{|e B|} \gg g T$. This parametric form is derived from one self-energy insertion to the fermion propagators. The leading behavior of the self-energy is $\sim g^{2} T$, while the propagator is of order $1 / \Delta \varepsilon \sim T /|e B|$. Thus, the combination of these factors leads to $\left(g^{2} T\right)(T /|e B|)=g^{2} T^{2} /|e B| \ll 1$.

Kinetic equations. - Next, we calculate the longitudinal conductivity of our main interest. To this end we must deal with the resummation over pinching singularities (see Ref. [15], for example). An efficient approach to resum higher order diagrams is solving the Bethe-Salpeter equations, as illustrated in Fig. 1, which amounts to the common formalism used in Ref. [16].

The Bethe-Salpeter equations can be translated to the linearized kinetic or Boltzmann equations as

$$
\begin{aligned}
2 P_{p}^{\mu}\left(\partial_{\mu}+q_{f} F_{\nu \mu} \partial_{p_{\nu}}\right) f_{p} & =-C[f], \\
2 \bar{P}_{p^{\prime}}^{\mu}\left(\partial_{\mu}-q_{f} F_{\nu \mu} \partial_{p_{\nu}^{\prime}}\right) \bar{f}_{p^{\prime}} & =-\bar{C}[f], \\
2 k^{\mu} \partial_{\mu} g_{k} & =-\tilde{C}[f]
\end{aligned}
$$

for quarks, antiquarks, and gluons, respectively, where $\partial_{p_{\nu}}:=\partial / \partial p_{\nu}$ and $C[f], \quad \bar{C}[f]$, and $\tilde{C}[f]$ represent the collision terms. In the above, $2 P_{p}^{\mu}:=\bar{u}(p) \gamma^{\mu} u(p)$ and $2 \bar{P}_{p^{\prime}}^{\mu}:=\bar{v}\left(p^{\prime}\right) \gamma^{\mu} v\left(p^{\prime}\right)$ with the wave functions $u(p)$ and $v\left(p^{\prime}\right)$ for particle and antiparticle, respectively, and the subscript $p, p^{\prime}$, and $k$ represent not only the momenta but also the Landau level $n$, the angular momentum $l$, the spin $s$, the color $c$, and the flavor $f$ collectively.

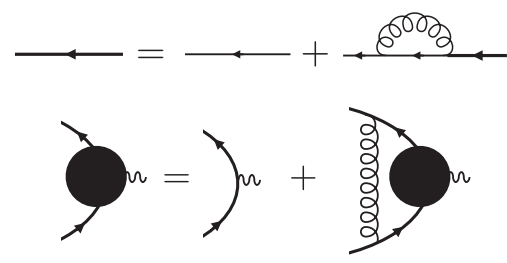

FIG. 1. Bethe-Salpeter equations: the resummed propagator with self-energy insertions (top) and the resummed vertex with ladder diagrams (bottom). 
To solve the Boltzmann equation perturbatively, we expand the distribution functions in terms of small deviations, $\delta f_{p}, \delta \bar{f}_{p}$, and $\delta g_{k}$, around the thermal equilibrium, $f_{\text {eq }}(p)=n_{F}\left(\varepsilon_{f n}-\mu\right), \bar{f}_{\text {eq }}(p)=n_{F}\left(\varepsilon_{f n}+\mu\right)$, and $g_{\text {eq }}(k)=$ $n_{B}\left(\omega_{k}\right)$, where $n_{B}$ is the Bose-Einstein distribution function and $\omega_{k}=|\boldsymbol{k}|$ is the energy of massless gluons. It would be convenient to introduce $\chi_{p}, \bar{\chi}_{p^{\prime}}$, and $\tilde{\chi}_{k}$ rescaled by common factors as $\delta f_{p}=\beta f_{\text {eq }}(p)\left[1-f_{\text {eq }}(p)\right] E_{z} \chi_{p}, \delta \bar{f}_{p^{\prime}}=$ $\beta \bar{f}_{\mathrm{eq}}\left(p^{\prime}\right)\left[1-\bar{f}_{\mathrm{eq}}\left(p^{\prime}\right)\right] E_{z} \bar{\chi}_{p^{\prime}}, \quad$ and $\quad \delta g_{k}=\beta g_{\mathrm{eq}}(k)[1+$ $\left.g_{\text {eq }}(k)\right] E_{z} \tilde{\chi}_{k}$, where $\beta=1 / T$ is the inverse temperature.

Suppose that we solved $\chi_{p}, \bar{\chi}_{p^{\prime}}$, and $\tilde{\chi}_{k}$ from the kinetic equations (6); we can express the electric current as $j_{z}=\sigma_{\|} E_{z}=\oiint_{p} 2 P_{p}^{3} q_{f}\left(\delta f_{p}-\delta \bar{f}_{p}\right)$, from which we can read $\sigma_{\|}$, where $\Varangle$ denotes the phase space sum of all quantum numbers and the invariant integration of momentum. In this way we come by the following formula

$$
\begin{aligned}
\sigma_{\|}= & \beta N_{\mathrm{c}} \sum_{f} \frac{q_{f}\left|q_{f} B\right|}{2 \pi} \sum_{n=0}^{\infty} \alpha_{n} \int \frac{d p_{z}}{2 \pi} \frac{p_{z}}{\varepsilon_{f n}} \\
& \times\left\{f_{\mathrm{eq}}(p)\left[1-f_{\mathrm{eq}}(p)\right] \chi_{p}-\bar{f}_{\mathrm{eq}}(p)\left[1-\bar{f}_{\mathrm{eq}}(p)\right] \bar{\chi}_{p}\right\} .
\end{aligned}
$$

Here, we introduced the spin degeneracy factor $\alpha_{n}$ as $\alpha_{0}=1$ and $\alpha_{n>0}=2$.

Now, let us return to our problem of solving Eq. (6). In the left-hand side, $\partial_{0}$ on $f_{\text {eq }}$ picks up a term $\propto \partial_{0} u_{z}$, where $u_{z}$ is the $z$ component of fluid velocity, which can be eliminated by the leading order hydrodynamic equation $\partial_{0} u_{z}=n_{e} E_{z} /\left(\mathcal{E}+\mathcal{P}_{z}\right)$. Then, the left-hand side of the first equation for quarks simplifies as

$2 P_{p}^{0}\left(\partial_{0}+q_{f} E_{z} \partial_{p_{z}}\right) f_{p}=-\beta W_{p} E_{z}\left(q_{f} \frac{p_{z}}{\varepsilon_{f n}}-\frac{n_{e} p_{z}}{\mathcal{E}+\mathcal{P}_{z}}\right)$.

Here, we defined $W_{p}:=2 P_{p}^{0} f_{\text {eq }}(p)\left[1-f_{\text {eq }}(p)\right]$. The second kinetic equation for $\bar{f}_{p}$ has the same structure as above with $f_{p}, W_{p}$, and $q_{f}$ replaced with $\bar{f}_{p}, \bar{W}_{p}$ := $2 P_{p}^{0} \bar{f}_{\mathrm{eq}}(p)\left[1-\bar{f}_{\mathrm{eq}}(p)\right]$, and $-q_{f}$. Likewise, the gluon equation is $2 \omega_{k} \partial_{0} g_{k}=-\beta \tilde{W}_{k}\left(-k_{z} \partial_{0} u_{z}\right)$ with $\tilde{W}_{k}:=$ $2 \omega_{k} g_{\mathrm{eq}}(k)\left[1+g_{\mathrm{eq}}(k)\right]$.

Using the following multicomponent symbols

$$
\mathcal{J}^{\mu}:=q_{f}\left(\begin{array}{c}
p^{\mu} / \varepsilon_{f n} \\
-p^{\prime \mu} / \varepsilon_{f n^{\prime}} \\
0
\end{array}\right), \quad \mathcal{T}^{0 \mu}:=\left(\begin{array}{c}
p^{\mu} \\
p^{\prime \mu} \\
k^{\mu}
\end{array}\right)
$$

we can summarize three kinetic equations as

$$
\mathcal{S}:=\mathcal{J}^{z}-\frac{n_{e} \mathcal{T}^{0 z}}{\mathcal{E}+\mathcal{P}_{z}}=\mathcal{L} \chi
$$

where the left-hand side will be denoted by $\mathcal{S}$ below, and the right-hand side represents the collision terms; $\mathcal{L}$ is a linear operator defined by

$$
\mathcal{L} \chi:=\mathcal{L}\left(\begin{array}{c}
\chi_{p} \\
\bar{\chi}_{p^{\prime}} \\
\tilde{\chi}_{k}
\end{array}\right)=\frac{1}{\beta E_{z}}\left(\begin{array}{c}
C[f] / W_{p} \\
\bar{C}[f] / \bar{W}_{p^{\prime}} \\
\tilde{C}[f] / \tilde{W}_{k}
\end{array}\right) .
$$

We should then solve $\chi=\mathcal{L}^{-1} \mathcal{S}$ using our symbolic notation. We note that $\mathcal{L}$ contains five zero eigenvalues (for a single flavor and more for multiflavors) with the eigenvectors $\mathcal{C}^{a}=\left\{\mathcal{J}^{0}, \mathcal{T}^{0 \mu}\right\}$ corresponding to the charge and the energy-momentum conservations. For two flavors $\mathcal{C}^{a}$ also contains the quark number conservation.

To formulate the projection procedure, let us introduce an inner product for two functions $A=\left(a_{p}, \bar{a}_{p^{\prime}}, \tilde{a}_{k}\right)$ and $B=\left(b_{p}, \bar{b}_{p^{\prime}}, \tilde{b}_{k}\right)$ by $(A, B):=\int_{p} W_{p} a_{p} b_{p}+\int_{p^{\prime}} \bar{W}_{p^{\prime}} \bar{a}_{p^{\prime}} \bar{b}_{p^{\prime}}+$ $\int_{k} \tilde{W}_{k} \tilde{a}_{k} \tilde{b}_{k}$. It is easy to rewrite Eq. (7) as $\sigma_{\|}=\beta\left(\mathcal{J}^{z}, \chi\right)$ using Eq. (9). With the zero eigenvectors $\mathcal{C}$ and the inner product, we define a projection operator onto functional space excluding zero eigenvalues as $\mathcal{Q} O:=O-$ $\sum_{a, b} \mathcal{C}^{a}(\mathcal{C}, \mathcal{C})_{a b}^{-1}\left(\mathcal{C}^{b}, O\right)$, where $(\mathcal{C}, \mathcal{C})_{a b}^{-1}$ is the inverse matrix of $\left(\mathcal{C}^{a}, \mathcal{C}^{b}\right)$. We see $\mathcal{Q}^{2}=\mathcal{Q}$ and $\mathcal{Q C}^{a}=0$ by construction. Using alternative expressions for the charge density and the enthalpy, i.e., $n_{e}=\beta\left(\mathcal{T}^{0 z}, \mathcal{J}^{z}\right)$ and $\mathcal{E}+\mathcal{P}_{z}=\beta\left(\mathcal{T}^{0 z}, \mathcal{T}^{0 z}\right)$ [17], we can write $\mathcal{S}=\mathcal{Q J}^{z}$. Noting $\mathcal{L}=\mathcal{L} \mathcal{Q}$, we find the formal solution of $\mathcal{L} \chi=\mathcal{S}$ as $\chi=\mathcal{Q} \mathcal{L}^{-1} \mathcal{Q S}$, where $\mathcal{Q} \mathcal{L}^{-1} \mathcal{Q}$ satisfies a relation $\mathcal{L} \mathcal{Q} \mathcal{L}^{-1} \mathcal{Q}=\mathcal{Q}$. We eventually obtain

$$
\sigma_{\|}=\beta\left(\mathcal{J}^{z}, \mathcal{Q} \mathcal{L}^{-1} \mathcal{Q S}\right)=\beta\left(\mathcal{S}, \mathcal{L}^{-1} \mathcal{S}\right) .
$$

This means the zero modes of $\mathcal{L}$ are already projected out once applied on $\mathcal{S}$.

Collision terms.-The collision term is the most complicated part. A strong magnetic field, $\sqrt{e B} \gg g T$, makes $1 \leftrightarrow 2$ processes such as the synchrotron radiation more enhanced than other ordinary processes by $e B /(g T)^{2} \gg 1$. That is, the $1 \leftrightarrow 2$ process of typical scale $\sim g^{2} e B / T^{2}$ is much greater than the $2 \leftrightarrow 2$ process of typical scale $\sim g^{4} \ln 1 / g$. For the $1 \leftrightarrow 2$ process there are three distinct contributions, $\quad C[f]=C_{q \rightarrow q g}[f]+C_{q g \rightarrow q}[f]+C_{q \bar{q} \rightarrow g}[f]$, where the subscripts represent processes illustrated in Fig. 2. We can also consider similar decompositions for $\bar{C}$ for antiquarks and $\tilde{C}$ for gluons.

After tedious calculations we find that the scattering amplitudes of the synchrotron radiation and the pair annihilation processes, $i \mathcal{M}_{p \rightarrow k+p^{\prime}}=i g \bar{u}\left(p^{\prime}\right) \gamma^{\mu} t_{a} u(p) \varepsilon_{\mu}^{*}(k)$ and $i \mathcal{M}_{p+p^{\prime} \rightarrow k}=i g \bar{v}\left(p^{\prime}\right) \gamma^{\mu} t_{a} u(p) \varepsilon_{\mu}^{*}(k)$, can be squared with the summation over the quantum numbers and the phase space, leading to 


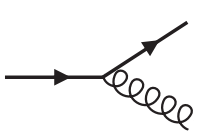

(a1) $q \rightarrow q g$

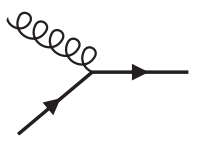

(a2) $q g \rightarrow q$

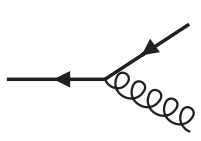

(b1) $\bar{q} \rightarrow \bar{q} g$

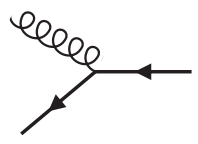

(b2) $\bar{q} g \rightarrow \bar{q}$

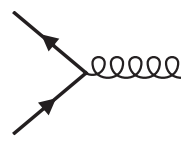

(c1) $q \bar{q} \rightarrow g$

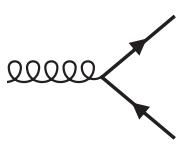

(c2) $g \rightarrow q \bar{q}$

FIG. 2. Diagrams of the synchrotron radiation process with a quark (a1), with an antiquark (b1), and with the pair annihilation (c1). Their inverse processes are (a2), (b2), and (c2), respectively.

$$
\begin{aligned}
& \int_{k, p, p^{\prime}}\left|\mathcal{M}_{p \rightarrow p^{\prime}+k}\right|^{2}(2 \pi)^{4} \delta^{(4)}\left(k-p+p^{\prime}\right) \\
& =-\frac{1}{2} \sum_{f, n>n^{\prime}} \int \frac{d p_{z}}{2 \pi} \frac{1}{2 \varepsilon_{f n}} \int_{p_{z-}^{\prime}}^{p_{z+}^{\prime}} \frac{d p_{z}^{\prime}}{2 \pi} \frac{1}{2 \varepsilon_{f n^{\prime}}} X\left(n, n^{\prime}, \xi_{-}^{f}\right), \\
& \int_{k, p, p^{\prime}}\left|\mathcal{M}_{p+p^{\prime} \rightarrow k}\right|^{2}(2 \pi)^{4} \delta^{(4)}\left(p+p^{\prime}-k\right) \\
& =\frac{1}{2} \sum_{f, n, n^{\prime}} \int \frac{d p_{z}}{2 \pi} \frac{1}{2 \varepsilon_{f n}} \int \frac{d p_{z}^{\prime}}{2 \pi} \frac{1}{2 \varepsilon_{f n^{\prime}}} X\left(n, n^{\prime}, \xi_{+}^{f}\right),
\end{aligned}
$$

where the allowed range of $p_{z}^{\prime}$ is restricted for the synchrotron radiation in Eq. (13) as $p_{z-}^{\prime}<p_{z}^{\prime}<p_{z+}^{\prime}$ with

$$
p_{z \pm}^{\prime}=p_{z} \frac{m_{f n}^{2}+m_{f n^{\prime}}^{2}}{2 m_{f n}^{2}} \pm \frac{m_{f n}^{2}-m_{f n^{\prime}}^{2}}{2 m_{f n}^{2}} \sqrt{m_{f n}^{2}+p_{z}^{2}} .
$$

Two integrands in Eqs. (13) and (14) are identical, i.e., $X\left(n, n^{\prime}, \xi_{k}\right):=g^{2} N_{\mathrm{c}} C_{F} \int\left[d^{2} p_{\perp} /(2 \pi)^{2}\right] \operatorname{tr}\left[\gamma_{\mu} S_{n}^{f}(p) \gamma^{\mu} S_{n^{\prime}}^{f}(p-k)\right]$ with a group factor $C_{F}:=\left(N_{\mathrm{c}}^{2}-1\right) /\left(2 N_{\mathrm{c}}\right)$, except for the kinematical constraint; that is, the argument of $X\left(n, n^{\prime}, \xi_{ \pm}^{f}\right)$ is given by

$$
\xi_{ \pm}^{f}=\frac{\left(\varepsilon_{f n} \pm \varepsilon_{f n^{\prime}}\right)^{2}-\left(p_{z} \pm p_{z}^{\prime}\right)^{2}}{2\left|q_{f} B\right|}
$$

Using Eq. (3) and properties of the Laguerre polynomials we find

$$
\begin{aligned}
X\left(n, n^{\prime}, \xi\right)= & g^{2} N_{\mathrm{c}} C_{F} \frac{\left|q_{f} B\right|}{2 \pi} e^{-\xi} \frac{n !}{n^{\prime} !} \xi^{n^{\prime}-n}\left\{\left[4 m_{f}^{2}\right.\right. \\
& \left.-4\left|q_{f} B\right|\left(n+n^{\prime}-\xi\right) \frac{1}{\xi}\left(n+n^{\prime}\right)\right] F\left(n, n^{\prime}, \xi\right) \\
& \left.+16\left|q_{f} B\right| n^{\prime}\left(n+n^{\prime}\right) \frac{1}{\xi} L_{n}^{\left(n^{\prime}-n\right)}(\xi) L_{n-1}^{\left(n^{\prime}-n\right)}(\xi)\right\},
\end{aligned}
$$

$$
F\left(n, n^{\prime}, \xi\right):= \begin{cases}1, & (n=0), \\ {\left[L_{n}^{\left(n^{\prime}-n\right)}(\xi)\right]^{2}+\frac{n^{\prime}}{n}\left[L_{n-1}^{\left(n^{\prime}-n\right)}(\xi)\right]^{2},} & (n>0) .\end{cases}
$$

Recovery of the lowest Landau level approximation.-It would be an instructive check whether the LLLA result is correctly recovered in the limit of $e B \gg T^{2}$ (at $\mu=0$ ). Since the synchrotron radiation changes the Landau level, we can safely discard it. For the pair annihilation process, $X\left(n=0, n^{\prime}=0, \xi\right)$ given in Eq. (17) simplifies as $X\left(0,0, \xi_{+}^{f}\right)=4 m_{f}^{2} g^{2} N_{\mathrm{c}} C_{F}\left(\left|q_{f} B\right| / 2 \pi\right) e^{-\xi_{+}^{0}}$ with $\xi_{+}^{0}=$ $\left[\left(\sqrt{p_{z}^{2}+m_{f}^{2}}+\sqrt{p_{z}^{\prime 2}+m_{f}^{2}}\right)^{2}-\left(p_{z}+p_{z}^{\prime}\right)^{2}\right] /\left(2\left|q_{f} B\right|\right)$,

which is nothing but $\xi_{+}$in Eq. (16) with $n=n^{\prime}=0$. When $\left|q_{f} B\right|$ is much larger than any other scales, we can approximate $e^{-\xi_{+}^{0}} \approx 1$. Then, the linearized kinetic equations reduce to

$$
\begin{aligned}
& q_{f} N_{\mathrm{c}} \frac{\left|q_{f} B\right|}{2 \pi} \beta f_{\mathrm{eq}}(p)\left[1-f_{\mathrm{eq}}(p)\right] \frac{p_{z}}{\varepsilon_{f 0}}=4 m_{f}^{2} g^{2} N_{\mathrm{c}} C_{F} \\
& \quad \times \beta \frac{\left|q_{f} B\right|}{2 \pi} \frac{1}{4 \varepsilon_{f 0}} \int \frac{d p_{z}^{\prime}}{2 \pi} \frac{1}{2 \varepsilon_{f 0}^{\prime}} f_{\mathrm{eq}}(p) \bar{f}_{\mathrm{eq}}\left(p^{\prime}\right)\left[1+g_{\mathrm{eq}}(k)\right] \chi_{p},
\end{aligned}
$$

where $\varepsilon_{f 0}=\sqrt{p_{z}^{2}+m_{f}^{2}}$ and $\varepsilon_{f 0}^{\prime}=\sqrt{p_{z}^{\prime 2}+m_{f}^{2}}$. We need to keep the current quark mass $m_{f}$ that breaks chiral symmetry to make the above LLLA expression sensible. In contrast, we can safely neglect the thermal mass $m_{T} \sim$ $g T \ll T$ that does not break chiral symmetry, though the thermal mass is greater than the current quark mass. Here, we do not have to consider mixing terms with $\bar{\chi}_{p^{\prime}}$. In this special limit, $\mathcal{L}$ is not a matrix and the matrix inversion is unnecessary. Actually, we can easily solve the above kinetic equation to obtain $\chi_{p}$. Thanks to the charge conjugation symmetry, the solution for antiquarks is $\bar{\chi}_{p}=-\chi_{p}$. Summarizing them, we arrive at the LLLA result from Eq. (12) as

$$
\begin{aligned}
\sigma_{\|}= & \sum_{f} \frac{N_{\mathrm{c}} \beta}{g^{2} C_{F} m_{f}^{2}} q_{f}^{2} \frac{\left|q_{f} B\right|}{2 \pi} \int \frac{d p_{z}}{2 \pi} \frac{p_{z}^{2}}{\varepsilon_{f 0}} \\
& \times \frac{f_{\mathrm{eq}}(p)\left[1-f_{\mathrm{eq}}(p)\right]^{2}}{\int \frac{d p_{z}^{\prime}}{2 \pi} \frac{1}{\varepsilon_{f 0}^{\prime}} \bar{f}_{\mathrm{eq}}\left(p^{\prime}\right)\left[1+g_{\mathrm{eq}}(k)\right]},
\end{aligned}
$$

which is consistent with Ref. [7].

Numerical results and discussions.-Below we will show numerical results, for which we should write down the matrix elements of $\mathcal{L}$ as a phase space convolution of 


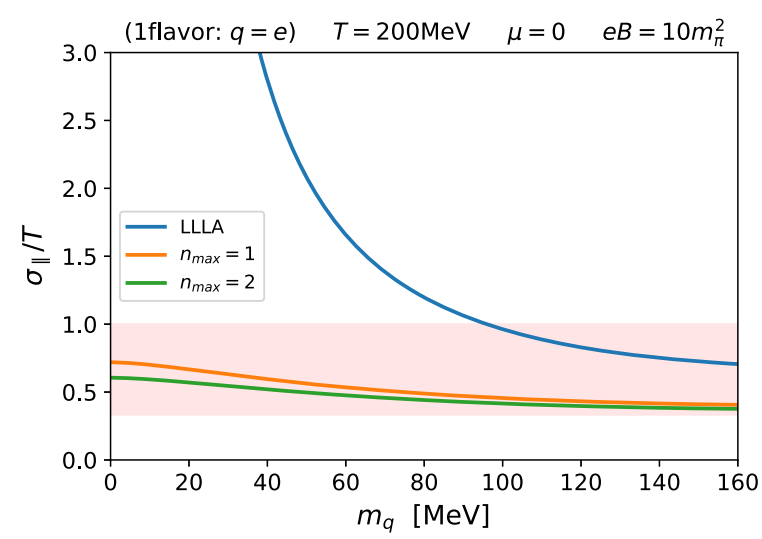

FIG. 3. Mass dependence of $\sigma_{\|}$for a single flavor at $T=200 \mathrm{MeV}, \mu=0, e B=10 m_{\pi}^{2}$, and $g^{2} /(4 \pi)=0.3$. The shaded region is the lattice-QCD estimate from Ref. [11].

$X\left(n, n^{\prime}, \xi_{ \pm}^{f}\right)$ and the distribution functions $f_{\text {eq }}, \bar{f}_{\text {eq }}$, and $g_{\text {eq }}$. Besides the flavor $f$ and the Landau level $n$, we should choose the complete set basis for functions of $p_{z}, k_{z}$, and $k_{\perp}$, which we will take the simplest polynomial form as $\hat{p}_{z}\left|p_{z}\right|^{m}$ for (anti)quarks and $\hat{k}_{z}\left|k_{z}\right|^{m} k_{\perp}^{l}$ for gluons with integral $m$ and $l$.

Figure 3 shows our numerical results for the current quark mass dependence of $\sigma_{\|} / T$ for a fictitious single flavor with $q=e$ at finite $T$ and $B$ but at zero $\mu$. We choose the QCD charge as $g^{2} /(4 \pi)=0.3$ [18]. We clearly see that the LLLA has artificial enhancement as $m_{q}$ approaches zero. For the numerical calculation we truncate the Landau level at $n_{\max }$. In the $e B=10 m_{\pi}^{2}$ case, the convergence of the Landau level sum is very fast and $n_{\max }=1$ already gives a good approximation, even though the LLLA badly breaks down in the small $m_{q}$ region. It is interesting that our result is quantitatively consistent with the lattice-QCD estimate $0.3 \leq \sigma / T \leq 1.0$ (for the quark charge squared sum $\left.C_{\mathrm{em}}=1\right)$ [11], which is indicated by the shaded region in Fig. 3.

The $B$ dependence of $\sigma_{\|} / T$ has a nonmonotonic structure as shown in Fig. 4, for which we adopted a physical

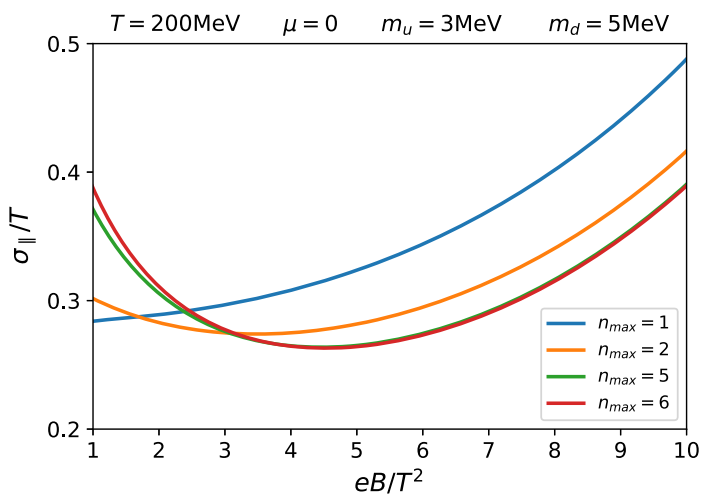

FIG. 4. Magnetic and $n_{\max }$ dependence of $\sigma_{\|} / T$.

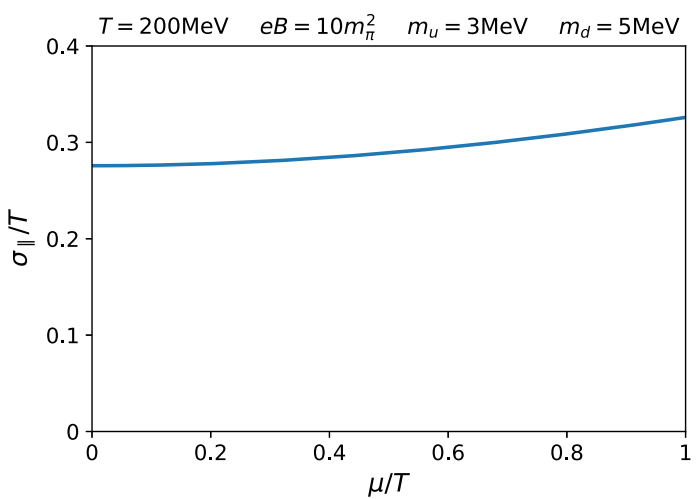

FIG. 5. Quark chemical potential dependence for $n_{\max }=2$.

parameter set with $u$ and $d$ quarks. For small $n_{\max }$ or strong $B$, the lowest Landau level contribution is dominant, and then $\sigma_{\|}$is linearly proportional to $B$ (reflecting the fact that the charge carrier increases), which explains the growing behavior at large $B$ in Fig. 4 . When $B$ is not so large, contributions from higher Landau levels lead to a larger interaction cross section due to the phase space factor, which pushes $\sigma_{\|}$down with larger $B$. As a result of the interplay of these competing effects, in an intermediate region of $B$, the increasing behavior of $\sigma_{\|}$looks quadratic; moreover, this nonmonotonic behavior is consistent with what is seen in the CME experiment in Ref. [3]. Although quantitative details may depend on the underlying theory, qualitative features should be the same for general physical systems (but could be different with different approximations, say, the relaxation time approximation [2] may lead to a different $B$ dependence).

Finally, we discuss the dependence of quark chemical potential $\mu$ as shown in Fig. 5. The carrier density is different from the net particle number but is the sum of particle and antiparticle numbers. This latter quantity is not changed by $\mu$, so $\sigma_{\|}$is rather insensitive to $\mu$.

In the future our estimated $B$ dependence of $\sigma_{\|}$could be tested by the lattice-QCD simulation at finite $B$, while our calculation at finite $\mu$ would be a unique prediction.

The authors thank Koichi Hattori, Daisuke Satow, and Misha Stephanov for useful comments and discussions. This work was supported by Japan Society for the Promotion of Science (JSPS) KAKENHI Grants No. 15H03652, No. 15K13479, No. 16K17716, and No. 17H06462.

[1] K. Fukushima, D. E. Kharzeev, and H. J. Warringa, Phys. Rev. D 78, 074033 (2008).

[2] D. T. Son and B. Z. Spivak, Phys. Rev. B 88, 104412 (2013).

[3] Q. Li, D. E. Kharzeev, C. Zhang, Y. Huang, I. Pletikosic, A. V. Fedorov, R. D. Zhong, J. A. Schneeloch, G. D. Gu, and T. Valla, Nat. Phys. 12, 550 (2016).

[4] L. McLerran and V. Skokov, Nucl. Phys. A929, 184 (2014).

[5] K. Tuchin, Phys. Rev. C 93, 014905 (2016). 
[6] K. Hattori and D. Satow, Phys. Rev. D 94, 114032 (2016).

[7] K. Hattori, S. Li, D. Satow, and H.-U. Yee, Phys. Rev. D 95, 076008 (2017).

[8] K. Fukushima, K. Hattori, H.-U. Yee, and Y. Yin, Phys. Rev. D 93, 074028 (2016).

[9] K. Hattori, X.-G. Huang, D. H. Rischke, and D. Satow, Phys. Rev. D 96, 094009 (2017).

[10] G. Aarts, C. Allton, J. Foley, S. Hands, and S. Kim, Phys. Rev. Lett. 99, 022002 (2007).

[11] H. T. Ding, A. Francis, O. Kaczmarek, F. Karsch, E. Laermann, and W. Soeldner, Phys. Rev. D 83, 034504 (2011).

[12] H.-T. Ding, O. Kaczmarek, and F. Meyer, Phys. Rev. D 94, 034504 (2016).
[13] D. N. Zubarev, A. V. Prozorkevich, and S. A. Smolyanskii, Theor. Math. Phys. 40, 821 (1979).

[14] V. Gusynin, V. Miransky, and I. Shovkovy, Phys. Lett. B 349, 477 (1995);.

[15] Y. Hidaka and T. Kunihiro, Phys. Rev. D 83, 076004 (2011).

[16] P. B. Arnold, G. D. Moore, and L. G. Yaffe, J. High Energy Phys. 11 (2000) 001.

[17] Y. Minami and Y. Hidaka, Phys. Rev. E 87, 023007 (2013).

[18] In the hard thermal loop calculation $g^{2} /(4 \pi)=0.326$ is assumed at $2 \pi T=1.5 \mathrm{GeV}$ [19]. Generally, fermionic processes without a Matsubara zero mode have better convergence, which is the case in our present calculation.

[19] N. Haque, A. Bandyopadhyay, J. O. Andersen, M. G. Mustafa, M. Strickland, and N. Su, J. High Energy Phys. 05 (2014) 027. 